\title{
A causal Schwarzschild-de Sitter interior solution by gravitational decoupling
}

\author{
L. Gabbanelli ${ }^{1, \mathrm{a}}$, J. Ovalle ${ }^{2,3, \mathrm{~b}}$, A. Sotomayor ${ }^{4, \mathrm{c}}$, Z. Stuchlik ${ }^{2, \mathrm{~d}}$, R. Casadio ${ }^{5,6, \mathrm{e}}$ \\ ${ }^{1}$ Departament de Física Quàntica i Astrofísica, Institut de Ciències del Cosmos (ICCUB), Universitat de Barcelona, Martí i Franquès 1, \\ 08028 Barcelona, Spain \\ ${ }^{2}$ Institute of Physics and Research Centre of Theoretical Physics and Astrophysics, Faculty of Philosophy and Science, Silesian University \\ in Opava, 74601 Opava, Czech Republic \\ ${ }^{3}$ Departamento de Física, Universidad Simón Bolívar, AP 89000, Caracas 1080, Venezuela \\ ${ }^{4}$ Departamento de Matemáticas, Universidad de Antofagasta, Antofagasta, Chile \\ 5 Dipartimento di Fisica e Astronomia, Alma Mater Università di Bologna, via Irnerio 46, 40126 Bologna, Italy \\ ${ }^{6}$ Istituto Nazionale di Fisica Nucleare, Sezione di Bologna, I.S. FLAG, viale Berti Pichat 6/2, 40127 Bologna, Italy
}

Received: 24 April 2019 / Accepted: 3 June 2019 / Published online: 11 June 2019

(C) The Author(s) 2019

\begin{abstract}
We employ the minimal geometric deformation approach to gravitational decoupling (MGD-decoupling) in order to build an exact anisotropic version of the Schwarzschild interior solution in a space-time with cosmological constant. Contrary to the well-known Schwarzschild interior, the matter density in the new solution is not uniform and possesses subluminal sound speed. It therefore satisfies all standard physical requirements for a candidate astrophysical object.
\end{abstract}

\section{Introduction}

The Schwarzschild interior metric is one of the best known solutions of Einstein's field equations [1]. This exact solution represents an isotropic self-gravitating object of uniform density (incompressible fluid), and has been widely studied, generally without considering the cosmological constant. As far as we know, it is one of the few analytic solutions for a bounded distribution which fits smoothly with the Schwarzschild exterior metric [2]. However, it cannot be used to represent a stellar model as its speed of sound is not subliminal (see Ref. [3] where a model of two fluids is used to circumvent the problem of causality). Despite of the above, some studies on the possible impact of the vacuum energy on perfect fluids have been carried out which made

\footnotetext{
a e-mail: gabbanelli@icc.ub.edu

b e-mail: jovalle@usb.ve

c e-mail: adrian.sotomayor@uantof.cl

d e-mail: zdenek.stuchlik@fpf.slu.cz

e e-mail: casadio@bo.infn.it
}

use of this solution for both positive and negative values of the cosmological constant. Such analyses can help to elucidate some properties of the Schwarzschild-(anti-)de Sitter spacetime in presence of matter [4,5] (see also [6-8]). Moreover, some alternatives to black holes, like the gravastars $[9,10]$, are mainly generated from this solution $[11,12]$, and an exact time-dependent version was recently reported in Ref. [13]. Therefore, it is not only natural, but also useful, to construct a possible extension of this solution for more realistic stellar scenarios, such as that represented by non-uniform and anisotropic self-gravitating objects. Above all, it would be very important to develop versions that do not suffer from the causal problem. However, given the complexity of Einstein's field equations, we know that extending a known solution to more complex scenarios is an arduous and difficult task [14], even more so if we wish to keep it physically acceptable. Fortunately, the so-called method of gravitational decoupling by Minimal Geometric Deformation (MGD-decoupling, henceforth) $[15,16]$, which has been widely used recently [17-32], has proved to be a powerful method to extend known solutions into more complex scenarios.

The original version of the MGD approach was developed in Refs. $[33,34]$ in the context of the brane-world scenario $[35,36]$, and it was eventually extended to study black hole solutions in Refs. [37,38] (for some earlier works on the MGD, see for instance Refs. [39-42], and Refs. [43-52] for some recent applications). On the other hand, the MGDdecoupling has three main characteristics that make it particularly useful in the search for new solutions of Einstein's field equations, namely: 
I. one can extend simple solutions of the Einstein equations into more complex domains. In fact, we can start from a source with energy-momentum tensor $\hat{T}_{\mu \nu}$ for which the metric is known and add the energy-momentum tensor of a second source,

$\hat{T}_{\mu \nu} \rightarrow T_{\mu \nu}=\hat{T}_{\mu \nu}+T_{\mu \nu}^{(1)}$.

We can then repeat the process with more sources $T_{\mu \nu}^{(i)}$ to extend the solution of the Einstein equations associated with the gravitational source $\hat{T}_{\mu \nu}$ into the domain of more intricate forms of gravitational sources $T_{\mu \nu}$;

II. one can also reverse the previous procedure in order to find a solution to Einstein's equations with a complex energy-momentum tensor $T_{\mu \nu}$ by splitting it into simpler components,

$T_{\mu \nu} \rightarrow \hat{T}_{\mu \nu}+T_{\mu \nu}^{(i)}$,

and solve Einstein's equations for each one of these components. Hence, we will have as many solutions as the components in the original energy-momentum tensor $T_{\mu \nu}$. Finally, by a simple combination of all these solutions, we will obtain the solution to the Einstein equations associated with the original energy-momentum tensor $T_{\mu \nu}$. We emphasise that the MGD-decoupling works as long as the sources do not exchange energy-momentum directly among them, to wit

$\nabla_{\mu} \hat{T}^{\mu \nu}=\nabla_{\mu} T^{(1) \mu \nu}=\cdots=\nabla_{\mu} T^{(n) \mu \nu}=0$,

which further clarifies that their interaction is purely gravitational;

III. it can be applied to theories beyond general relativity. For instance, given the modified action [16]

$S_{\mathrm{G}}=S_{\mathrm{EH}}+S_{\mathrm{X}}=\int\left[\frac{R}{2 k^{2}}+\mathcal{L}_{\mathrm{M}}+\mathcal{L}_{\mathrm{X}}\right] \sqrt{-g} d^{4} x$,

where $\mathcal{L}_{\mathrm{M}}$ contains all matter fields in the theory and $\mathcal{L}_{\mathrm{X}}$ is the Lagrangian density of a new gravitational sector with an associated (effective) energy-momentum tensor

$\theta_{\mu \nu}=\frac{2}{\sqrt{-g}} \frac{\delta\left(\sqrt{-g} \mathcal{L}_{\mathrm{X}}\right)}{\delta g^{\mu \nu}}=2 \frac{\delta \mathcal{L}_{\mathrm{X}}}{\delta g^{\mu \nu}}-g_{\mu \nu} \mathcal{L}_{\mathrm{X}}$,

the method in I. allows one to extend all the known solutions of the Einstein-Hilbert action $S_{\mathrm{EH}}$ into the domain of modified gravity represented by $S_{\mathrm{G}}$. This represents a straightforward way to study the consequences of extended gravity on general relativity.
In this paper we will apply the procedure I. to the Schwarzschild interior solution in order to build a new interior configuration with non-uniform matter density and anisotropic pressure.

The paper is organised as follows: in Sect. 2, we start from the Einstein equations with cosmological constant for a spherically symmetric stellar distribution and we show how to decoupling two spherically symmetric and static gravitational sources $\left\{T_{\mu \nu}, \theta_{\mu \nu}\right\}$. After providing details on the matching conditions at the star surface under the MGDdecoupling, in Sect. 3, we implement the MGD-decoupling following the scheme I. to generate the extended version of the Schwarzschild solution; finally, we summarise our conclusions in Sect. 4.

\section{Spherically symmetric stellar distribution}

Let us start from the standard Einstein field equations ${ }^{1}$

$R_{\mu \nu}-\frac{1}{2} R g_{\mu \nu}+\Lambda g_{\mu \nu}=k^{2} T_{\mu \nu}$,

where $\Lambda$ is a positive cosmological constant. The energymomentum tensor $T_{\mu \nu}$ in Eq. (2.1) is given by

$T_{\mu \nu}=\hat{T}_{\mu \nu}+\theta_{\mu \nu}$,

where $\hat{T}_{\mu \nu}$ represents the energy-momentum tensor of a perfect fluid, and $\theta_{\mu \nu}$ adds anisotropic effects on $T_{\mu \nu}$. Since the Einstein tensor is divergence free, the total energy momentum tensor $T_{\mu \nu}$ must satisfy the conservation equation

$\nabla_{v} T^{\mu \nu}=0$.

In Schwarzschild-like coordinates, the spherically symmetric metric reads

$d s^{2}=e^{\nu(r)} d t^{2}-e^{\lambda(r)} d r^{2}-r^{2}\left(d \theta^{2}+\sin ^{2} \theta d \phi^{2}\right)$,

where $v=v(r)$ and $\lambda=\lambda(r)$ are functions of the areal radius $r$ only, ranging from $r=0$ (the star's centre) to some $r=R>0$ (the star's surface). The cosmological constant can be thought to contribute the stress-energy tensor being responsible for the expansion of the universe, with a nonzero vacuum energy density and negative pressure satisfying $\rho_{\text {vac }}=-p_{\text {vac }}=\Lambda / k^{2}$. Explicitly, the field equations read

$$
\begin{aligned}
& k^{2}\left(\rho+\theta_{0}^{0}\right)+\Lambda=\frac{1}{r^{2}}-e^{-\lambda}\left(\frac{1}{r^{2}}-\frac{\lambda^{\prime}}{r}\right), \\
& k^{2}\left(-p+\theta_{1}^{1}\right)+\Lambda=\frac{1}{r^{2}}-e^{-\lambda}\left(\frac{1}{r^{2}}+\frac{\nu^{\prime}}{r}\right),
\end{aligned}
$$

\footnotetext{
${ }^{1}$ We use the metric signature (+- - ) and the constant $k^{2}=8 \pi G_{\mathrm{N}}$.
} 


$$
\begin{aligned}
& k^{2}\left(-p+\theta_{2}^{2}\right)+\Lambda \\
& \quad=\frac{1}{4} e^{-\lambda}\left[-2 v^{\prime \prime}-v^{\prime 2}+\lambda^{\prime} v^{\prime}-2 \frac{v^{\prime}-\lambda^{\prime}}{r}\right],
\end{aligned}
$$

while the conservation equation, which is a linear combination of Eqs. (2.5)-(2.7), yields

$$
-p^{\prime}-\frac{v^{\prime}}{2}(\rho+p)+\left(\theta_{1}^{1}\right)^{\prime}-\frac{v^{\prime}}{2}\left(\theta_{0}^{0}-\theta_{1}^{1}\right)-\frac{2}{r}\left(\theta_{2}^{2}-\theta_{1}^{1}\right)=0,
$$

where $f^{\prime} \equiv \partial_{r} f$.

By simple inspection of Eqs. (2.5)-(2.7), we can identify an effective density

$\tilde{\rho}=\rho+\theta_{0}^{0}$,

an effective isotropic pressure

$\tilde{p}_{r}=p-\theta_{1}^{1}$,

and an effective tangential pressure

$\tilde{p}_{t}=p-\theta_{2}^{2}$.

This clearly illustrate that the source $\theta_{\mu \nu}$ generates an anisotropy

$\Pi \equiv \tilde{p}_{t}-\tilde{p}_{r}=\theta_{1}^{1}-\theta_{2}^{2}$

inside the stellar distribution.

Eqs. (2.5)-(2.7) contain five unknown functions, namely, three physical variables: the density $\tilde{\rho}(r)$, the radial pressure $\tilde{p}_{r}(r)$ and the tangential pressure $\tilde{p}_{t}(r)$; and two geometric functions: the temporal metric function $v(r)$ and the radial metric function $\lambda(r)$. Therefore these equations form an indefinite system $[53,54]$ which requires additional information to produce any specific solution.

\subsection{Gravitational decoupling by MGD}

In order to solve the Einstein Eqs. (2.5)-(2.8) we implement the MGD-decoupling. In this approach, one starts from a solution for the isotropic fluid and the field equations with the anisotropic source $\theta_{\mu \nu}$ will take the form of effective "quasi-Einstein" equations [see Eqs. (2.22)-(2.24) below].

A solution to Eqs. (2.5)-(2.8) with $\theta_{\mu \nu}=0$ will be given by a General Relativity perfect fluid solution with cosmological constant $\Lambda$ and be characterised by the four functions $\{\xi, \mu, \rho, p\}$ such that the metric reads

$d s^{2}=e^{\xi(r)} d t^{2}-e^{\mu(r)} d r^{2}-r^{2}\left(d \theta^{2}+\sin ^{2} \theta d \phi^{2}\right)$,

where $e^{-\mu(r)} \equiv 1-\frac{k^{2}}{r} \int_{0}^{r} x^{2} \rho d x=1-\frac{2 m(r)}{r}$

is the standard General Relativity expression containing the Misner-Sharp mass function $m=m(r)$. Next, we turn on the anisotropic effects by adding the $\theta_{\mu \nu}$. These effects can be encoded in the geometric deformation undergone by the perfect fluid geometry in Eq. (2.13), namely

$$
\begin{gathered}
\xi \mapsto v=\xi+\alpha g, \\
e^{-\mu} \mapsto e^{-\lambda}=e^{-\mu}+\alpha f,
\end{gathered}
$$

where $g$ and $f$ are the deformations undergone by the temporal and radial metric component of the perfect fluid geometry $\{\xi, \mu\}$, respectively. Among all possible deformations (2.15) and (2.16), the so-called minimal geometric deformation is given by $g=0$ and $f=f^{*}$, where $f^{*}$ satisfies a suitable condition $[33,34]$ in order to minimise the departure from General Relativity. Only the radial metric component therefore changes to

$e^{-\mu(r)} \mapsto e^{-\lambda(r)}=e^{-\mu(r)}+\alpha f^{*}(r)$.

The system (2.5)-(2.8) can be decoupled by plugging the deformation (2.17) into the Einstein equations (2.5)-(2.7). In fact, the system splits into two sets of equations: (i) one having the standard Einstein field equations for a perfect fluid with cosmological constant $\Lambda$, whose metric is given by Eq. (2.13) with $\xi(r)=v(r)$,

$$
\begin{aligned}
& k^{2} \rho+\Lambda=\frac{1}{r^{2}}-e^{-\mu}\left(\frac{1}{r^{2}}-\frac{\mu^{\prime}}{r}\right), \\
& k^{2}(-p)+\Lambda=\frac{1}{r^{2}}-e^{-\mu}\left(\frac{1}{r^{2}}+\frac{v^{\prime}}{r}\right), \\
& k^{2}(-p)+\Lambda \\
& =\frac{1}{4} e^{-\mu}\left[-2 v^{\prime \prime}-v^{\prime 2}+\mu^{\prime} v^{\prime}-2 \frac{v^{\prime}-\mu^{\prime}}{r}\right],
\end{aligned}
$$

along with the conservation equation (2.3) with $\theta_{\mu \nu}=0$, namely $\nabla_{v} \hat{T}^{\mu \nu}=0$, yielding

$p^{\prime}+\frac{\xi^{\prime}}{2}(\rho+p)=0$

which is a linear combination of Eqs (2.18)-(2.20); and (ii) one for the source $\theta_{\mu \nu}$, which reads

$$
\begin{aligned}
& k^{2} \theta_{0}^{0}=-\frac{\alpha f^{*}}{r^{2}}-\frac{\alpha f^{*^{\prime}}}{r}, \\
& k^{2} \theta_{1}^{1}=-\alpha f^{*}\left(\frac{1}{r^{2}}+\frac{v^{\prime}}{r}\right), \\
& k^{2} \theta_{2}^{2} \\
& =-\frac{\alpha f^{*}}{4}\left(2 v^{\prime \prime}+v^{\prime 2}+\frac{2 v^{\prime}}{r}\right)-\frac{\alpha f^{*^{\prime}}}{4}\left(v^{\prime}+\frac{2}{r}\right) .
\end{aligned}
$$


The conservation equation $\nabla_{v} \theta^{\mu \nu}=0$ explicitly reads

$\left(\theta_{1}^{1}\right)^{\prime}-\frac{v^{\prime}}{2}\left(\theta_{0}^{0}-\theta_{1}^{1}\right)-\frac{2}{r}\left(\theta_{2}^{2}-\theta_{1}^{1}\right)=0$,

which is a linear combination of Eqs. (2.22)-(2.24). We recall that, under these conditions, there is no exchange of energymomentum between the perfect fluid and the source $\theta_{\mu \nu}$ and therefore their interaction is purely gravitational.

\subsection{Matching conditions at the surface}

The interior $(0 \leq r \leq R)$ of the self-gravitating system of radius $(r=R)$ is described by the MGD metric

$d s^{2}=e^{v^{-}(r)} d t^{2}-\left[1-\frac{2 \tilde{m}(r)}{r}\right]^{-1} d r^{2}-r^{2}\left(d \theta^{2}+\sin ^{2} \theta d \phi^{2}\right)$,

where the interior mass function is given by

$\tilde{m}(r)=m(r)-\frac{r}{2} \alpha f^{*}(r)$,

with the Misner-Sharp mass $m$ given in Eq. (2.14) and $f^{*}$ the geometric deformation in Eq. (2.17). On the other hand, the exterior $(r>R)$ space-time will be described by the Schwarzschild-de Sitter metric

$$
\begin{aligned}
d s^{2}= & \left(1-\frac{2 \mathcal{M}}{r}-\frac{\Lambda}{3} r^{2}\right) d t^{2} \\
& -\frac{d r^{2}}{\left(1-\frac{2 \mathcal{N}}{r}-\frac{\Lambda}{3} r^{2}\right)}-r^{2}\left(d \theta^{2}+\sin ^{2} \theta d \phi^{2}\right) .
\end{aligned}
$$

The metrics in Eqs. (2.26) and (2.28) must satisfy the Israel-Darmois matching conditions [55] at the star surface $\Sigma$ defined by $r=R$. In particular, the continuity of the metric across $r=R$ implies

$e^{v^{-}(R)}=1-\frac{2 \mathcal{M}}{R}-\frac{\Lambda}{3} R^{2}$,

and

$1-\frac{2 M}{R}+\alpha f_{R}^{*}=1-\frac{2 \mathcal{M}}{R}$

where $M=m(R)$ and $f_{R}^{*}=f^{*}(R)$ is the minimal geometric deformation evaluated at the star surface.

Likewise, the extrinsic curvature (or second fundamental form) of spheres

$K_{\mu \nu}=\nabla_{\mu} r_{\nu}$ where $r_{\mu}$ is the unit radial vector normal to a surface of constant $r$, must be continuous across the sphere $\Sigma$, which can be written in terms of the Einstein tensor as ${ }^{2}$

$\left[G_{\mu \nu} r^{\nu}\right]_{\Sigma} \equiv \lim _{r \rightarrow R^{+}}\left(G_{\mu \nu} r^{\nu}\right)-\lim _{r \rightarrow R^{-}}\left(G_{\mu \nu} r^{\nu}\right)=0$

Using Eq. (2.32) and the general Einstein equations (2.1), we then find

$\left[\left(k^{2} T_{\mu \nu}+\Lambda g_{\mu \nu}\right) r^{\nu}\right]_{\Sigma}=0$

which leads to

$\left[k^{2}\left(p-\theta_{1}^{1}\right)-\Lambda\right]_{\Sigma}=0$.

This matching condition takes the final form

$p_{R}-\left(\theta_{1}^{1}\right)_{R}^{-}=0$,

where $p_{R} \equiv p(R)$ and $\left(\theta_{1}{ }^{1}\right)_{R}^{-} \equiv \theta_{1}{ }^{1}\left(r \rightarrow R^{-}\right)$. The condition (2.35) holds in general for the second fundamental form associated with the Einstein equations (2.1) and the energymomentum (2.2). After decoupling the source $\theta_{\mu \nu}$ and by using Eq. (2.23) for the interior geometry, Eq. (2.35) can be written as

$\tilde{p}_{R} \equiv p_{R}+\alpha \frac{f_{R}^{*}}{k^{2}}\left(\frac{1}{R^{2}}+\frac{v_{R}^{\prime}}{R}\right)=0$,

where $\left.v_{R}^{\prime} \equiv \partial_{r} v^{-}\right|_{r=R}$. Eqs. (2.29), (2.30) and (2.36) are the necessary and sufficient conditions for matching the interior MGD metric (2.26) with the outer Schwarzschild-de Sitter metric (2.28).

The expression in Eq. (2.36) in particular contains critical information about the conditions that the self-gravitating system must fulfil in order to be consistently coupled with the Schwarzschild-de Sitter geometry (2.28). First of all, the effective radial pressure $\tilde{p}$ at the surface must vanish, which is a very well-known result. However, if the geometric deformation $f^{*}(r<R)$ is positive, hence weakening the gravitational field, [see Eq. (2.27)], the exterior geometry (2.28) can only be compatible with a non-vanishing $\theta_{\mu \nu}$ if the perfect fluid has $p_{R}<0$, which may be interpreted as regular matter with a solid crust [43]. If we want to avoid having a solid-crust and keep the standard condition $p_{R}=0$, we must impose that the anisostropic effects on the radial pressure pressure vanish at $r=R$. For instance, this is achieved if we assume that $\left(\theta_{1}{ }^{1}\right)_{R}^{-} \sim p_{R}$ in Eq. (2.35), which leads to a vanishing inner deformation $f_{R}^{*}=0$ [see further Eq. (3.13)].

${ }^{2}$ For more details see for instance Ref. [56]. 


\section{Anisotropic Schwarzschild-de Sitter interior solution}

Let us recall that the interior Schwarzschild-de Sitter solution for the system (2.18)-(2.21) represents a stellar system of radius $R$ formed by an incompressible perfect fluid of total mass $M$ and is given by [6]

$$
\begin{aligned}
& e^{\nu(r)}=\left[A-B \sqrt{1-\frac{r^{2}}{C^{2}}}\right]^{2} \\
& e^{-\mu(r)}=1-\frac{r^{2}}{C^{2}} \\
& k^{2} \rho=\frac{3}{C^{2}}-\Lambda \\
& k^{2} p(r)=\frac{A\left(1-\Lambda C^{2}\right)-B\left(3-\Lambda C^{2}\right) \sqrt{1-\frac{r^{2}}{C^{2}}}}{C^{2}\left(B \sqrt{1-\frac{r^{2}}{C^{2}}}-A\right)} .
\end{aligned}
$$

The constants $A, B$ and $C$ can be expressed in terms of the physical parameters $M, R$ and $\Lambda$ by means of the matching conditions with the outer Schwarzschild-de Sitter vacuum (2.28). In fact, Eqs. (2.29), (2.30) and (2.36) with $\alpha=0$ yield $\mathcal{M}=M$ and

$$
\begin{aligned}
& A=B \frac{3-\Lambda C^{2}}{1-\Lambda C^{2}} \sqrt{1-\frac{R^{2}}{C^{2}}} \\
& B=\frac{3 M-\Lambda R^{3}}{6 M+\Lambda R^{3}} \\
& \frac{R^{2}}{C^{2}}=\frac{2 M}{R}+\frac{\Lambda R^{2}}{3} .
\end{aligned}
$$

We see that the pressure (3.4) becomes singular in the centre $r=0$ when

$$
A=B .
$$

We can avoid the singularity, while we keep $p(0)>0$, when the compactness satisfies

$\frac{M}{R}<\frac{1}{9}\left(2+\sqrt{4-3 \Lambda R^{2}}\right)$,

which is the Buchdahl inequality with cosmological constant (for the details, see for instance, Ref. [57]). For simplicity, we just show the final expressions for the case $\Lambda=0^{3}$, for which we have

$$
\begin{aligned}
& e^{\nu(r)}=\frac{1}{4}\left(\sqrt{1-\frac{2 M r^{2}}{R^{3}}}-3 \sqrt{1-\frac{2 M}{R}}\right)^{2} \\
& e^{-\mu(r)}=1-\frac{2 M r^{2}}{R^{3}}
\end{aligned}
$$

\footnotetext{
${ }^{3}$ From the phenomenological point of view, the cosmological constant $\Lambda \sim 10^{-52}$ appears irrelevantly small for any astrophysical systems.
}

$$
k^{2} p(r)=\frac{6 M\left(\sqrt{R^{3}-2 M r^{2}}-R \sqrt{R-2 M}\right)}{R^{3}\left(3 R \sqrt{R-2 M}-\sqrt{R^{3}-2 M r^{2}}\right)},
$$

from which we easily see that $p_{R}=0$, as required.

In order to generate the anisotropic version of the Eqs. (3.1)-(3.4), we need to determinate the anisotropic source $\theta_{\mu \nu}$, whose field equations are given by the expressions (2.22)-(2.24). This system has four unknowns and we therefore need to give some physically motivated prescriptions. Since we further want to avoid having a solid crust at the surface, we require the radial pressure satisfies the "mimic constraint" defined by

$\theta_{1}^{1}(r)=\alpha p(r)$

which, according to Eqs. (2.19) and (2.23), yields

$f^{*}(r)=-e^{-\mu(r)}+\frac{1-\Lambda r^{2}}{1+r v^{\prime}(r)}$.

We remark that the constraint (3.13) ensures that the effective radial pressure vanishes at $r=R$, since $\tilde{p}(R)=p(R)=0$ from Eq. (3.4).

Using the expression (3.14) in the MGD deformation (2.17), the radial metric component becomes

$$
\begin{aligned}
& e^{-\lambda(r)}=(1-\alpha) e^{-\mu(r)}+\alpha \frac{1-\Lambda r^{2}}{1+r v^{\prime}(r)} \\
& =\left(1-\frac{r^{2}}{C^{2}}\right) \\
& +\alpha \frac{r^{2}}{C^{2}}\left[\frac{A\left(1-\Lambda C^{2}\right)-B\left(3-\Lambda C^{2}\right) \sqrt{1-\frac{r^{2}}{C^{2}}}}{A \sqrt{1-\frac{r^{2}}{C^{2}}}-B\left(1-\frac{3 r^{2}}{C^{2}}\right)}\right] \\
& \sqrt{1-\frac{r^{2}}{C^{2}}},
\end{aligned}
$$

where the minimal geometric deformation is given by

$$
\begin{gathered}
f^{*}(r)=\frac{r^{2}}{C^{2}}\left[\frac{A\left(1-\Lambda C^{2}\right)-B\left(3-\Lambda C^{2}\right) \sqrt{1-\frac{r^{2}}{C^{2}}}}{A \sqrt{1-\frac{r^{2}}{C^{2}}}-B\left(1-\frac{3 r^{2}}{C^{2}}\right)}\right] \\
\sqrt{1-\frac{r^{2}}{C^{2}}} .
\end{gathered}
$$

By using the metric functions $v(r)$ and $\lambda(r)$ in (3.1) and (3.15) in the field equations (2.5)-(2.7), we find the effective density

$k^{2} \tilde{\rho}(r)=k^{2} \rho-\alpha\left(\frac{f^{*}(r)}{r^{2}}+\frac{f^{*^{\prime}}(r)}{r}\right)$, 
the effective radial pressure

$k^{2} \tilde{p}_{r}(r)=(1-\alpha) k^{2} p(r)$,

and the effective tangential pressure

$\tilde{p}_{t}(r)=\tilde{p}_{r}(r)+\Pi(\alpha, r)$,

where

$$
\begin{gathered}
\Pi(\alpha, r)=\alpha \frac{A\left(1-\frac{r^{2}}{C^{2}}\right)^{3 / 2}-B\left(1-\frac{2 r^{2}}{C^{2}}+\frac{2 r^{4}}{C^{4}}\right)}{k^{2} r^{2}\left(1-\frac{r^{2}}{C^{2}}\right)^{3 / 2}\left(B \sqrt{1-\frac{r^{2}}{C^{2}}}-A\right)} f^{*}(r) \\
+\alpha \frac{A \sqrt{1-\frac{r^{2}}{C^{2}}}-B\left(1-\frac{2 r^{2}}{C^{2}}\right)}{2 k^{2} r\left[A \sqrt{1-\frac{r^{2}}{C^{2}}}-B\left(1-\frac{r^{2}}{C^{2}}\right)\right]} f^{*^{\prime}}(r), \quad(3.20)
\end{gathered}
$$

and is rather cumbersome to display explicitly. The expressions (3.1), (3.15), (3.17)-(3.19) represent an exact solution for the anisotropic system (2.5)-(2.7).

We can next match the interior metric (2.4) with metric functions (3.1) and (3.15) with the exterior Schwarzschildde Sitter vacuum solution (2.28). We can see that, for a given total mass $M$ and radius $R$, we have four unknown parameters, namely $A, B$ and $C$ from the interior solution in Eqs. (3.1) and (3.15), and the mass $\mathcal{M}$ in Eq. (2.28). However, the mass $M$ is related to the constant $C$ and the radius $R$ by the definition (2.14). We therefore have only three unknown constants to be determined by the three conditions (2.29), (2.30) and (2.35) at the star surface. The continuity of the metric given by Eqs. (2.29) and (2.30) leads to

$$
\left(A-B \sqrt{1-\frac{R^{2}}{C^{2}}}\right)^{2}=1-\frac{2 \mathcal{M}}{R}-\frac{\Lambda}{3} R^{2},
$$

and

$$
\left(1-\frac{R^{2}}{C^{2}}\right)+\alpha f_{R}^{*}=1-\frac{2 \mathcal{M}}{R}-\frac{\Lambda}{3} R^{2},
$$

with $f_{R}^{*}=f^{*}(R)$ can be obtained from Eq. (3.16). Continuity of the second fundamental form in Eq. (2.35) under the constraint (3.13) now holds provided

$p_{R}=0$,

which is ensured by the same expression for $A$ shown in Eq. (3.5). This result is in agreement with our prescription to avoid having a solid crust and also ensures that

$$
f_{R}^{*}=0
$$

as we can verified from Eq. (3.16). Therefore the condition (3.22) leads to

$\mathcal{M}=M$,

where Eq. (3.3) has been used. The result in Eq. (3.25) is in agreement with the expressions in (2.30) and (3.24) and tells us that the the constant $C$ is also related to $M, R$ and $\Lambda$ by the same expression given in Eq. (3.7). We remark that the condition (3.24) shows that, despite the anisotropic effects, the total mass of the stellar distribution remains unchanged. Finally, by using Eqs. (3.5) and (3.25) in the matching condition (3.21), we obtain the same expression for the constant $B$ in Eq. (3.6). We therefore conclude that the relations between the constants $A, B$ and $C$ in terms of $M, R$ and $\Lambda$ are not affected by the anisotropy.

Given a stellar distribution of mass $M$ and radius $R$ in a background with cosmological constant $\Lambda$, we can now analyse the anisotropic effects on physical variables for different values of $\alpha$. The first thing to notice is that the effective radial pressure $\tilde{p}_{r}$ remains proportional to the isotropic expression (3.12), and we therefore find the usual Buchdahl limit for the star compactness [58] with cosmological constant in Eq. (3.9). This result is further supported by the fact that both the effective density $\tilde{\rho}$ and the tangential pressure $\tilde{p}$ do not show any singularity for $0 \leq r \leq R$ when Eq. (3.9) holds. Moreover, the effective density is not uniform and $\tilde{\rho}^{\prime}$ turns out to be proportional to $-\alpha$, which implies that the effective density decreases (increases) towards the surface for $\alpha>0$ (respectively, $\alpha<0$ ). In the following we shall therefore only consider cases with $\alpha>0$ so that the effective density decreases from the centre outwards.

Since the explicit expressions are rather cumbersome, Figure 1 shows the effective radial and tangential pressures in Eqs. (3.18) and (3.19) for two values of $\alpha>0$ (compared to the standard Schwarzschild solution $\alpha=0$ ). We see that the anisotropy decreases the effective radial pressures $\tilde{p}_{r}$ for all values of $0 \leq r \leq R$. The effective tangential pressure $\tilde{p}_{t}$ is always smaller than $\tilde{p}_{r}$ in the centre of the star, but decreases more slowly, and eventually becomes larger than $\tilde{p}_{r}$, near the surface. Moreover, the tangential pressure at the surface $\tilde{p}_{t}(R)>0$, whereas $\tilde{p}_{r}(R)=p_{R}=0$ by construction. On the other hand, we see from Figure 2 that the effective density $\tilde{\rho}$ in Eq. (3.17) also shows a similar behaviour to $\tilde{p}_{t}$, and the anisotropy $\Pi$ increases steadily from the centre (where $\Pi=0$ ) towards the surface. We want to remark that both the dominant energy condition,

$\tilde{\rho} \geq\left|\tilde{p}_{r}\right|, \quad \tilde{\rho} \geq\left|\tilde{p}_{t}\right|$,

and strong energy condition

$\tilde{\rho}+\tilde{p}_{r}+2 \tilde{p}_{t} \geq 0, \quad \tilde{\rho}+\tilde{p}_{r} \geq 0, \quad \tilde{\rho}+\tilde{p}_{t} \geq 0$ 


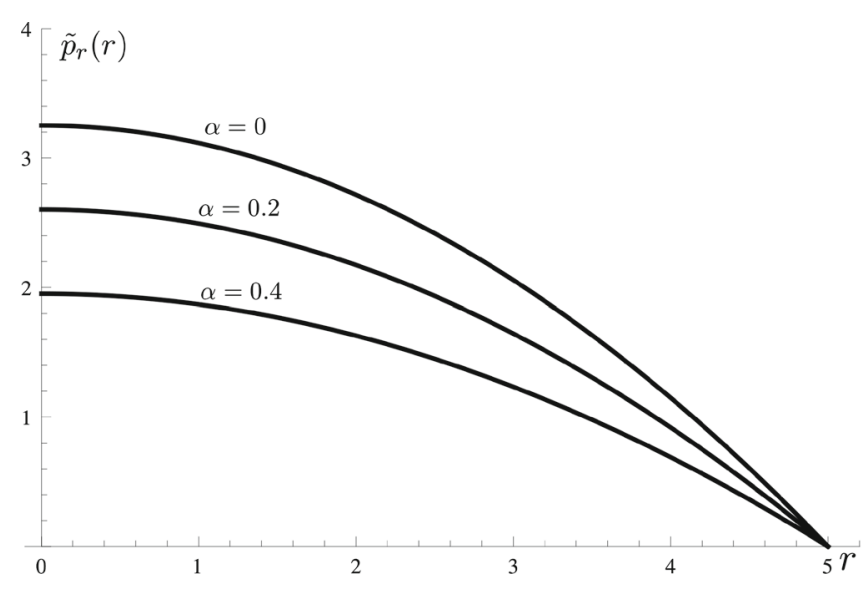

Fig. 1 Effective radial pressure $\tilde{p}_{r}(r)\left[10^{-4}\right]$ and effective tangential pressure $\tilde{p}_{t}(r)\left[10^{-4}\right]$ for a stellar system with compactness $M / R=0.2$ compared to the standard Schwarzschild case $\alpha=0$ with $p_{r}=p_{t}$.

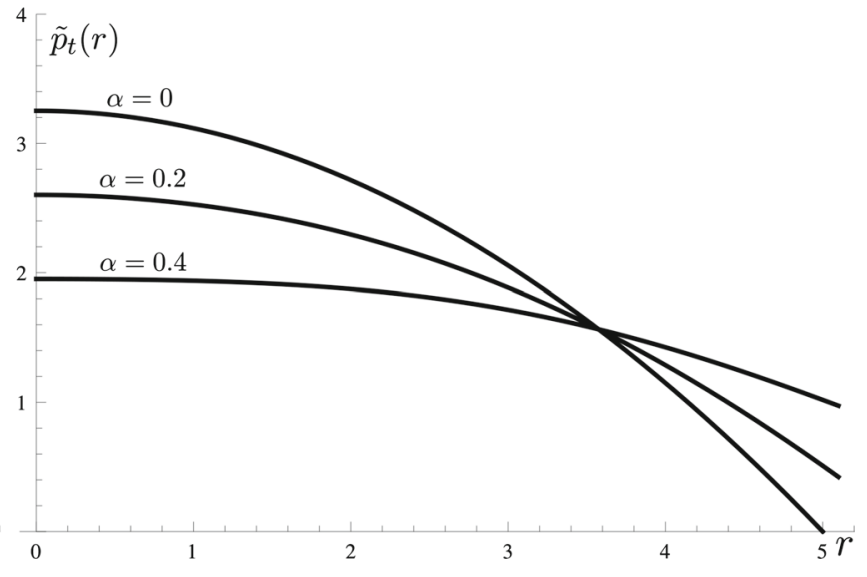

Radial coordinates are in units of $M(r=5=R)$. The cosmological constant $\Lambda \sim 10^{-52}$ is too small to be relevant

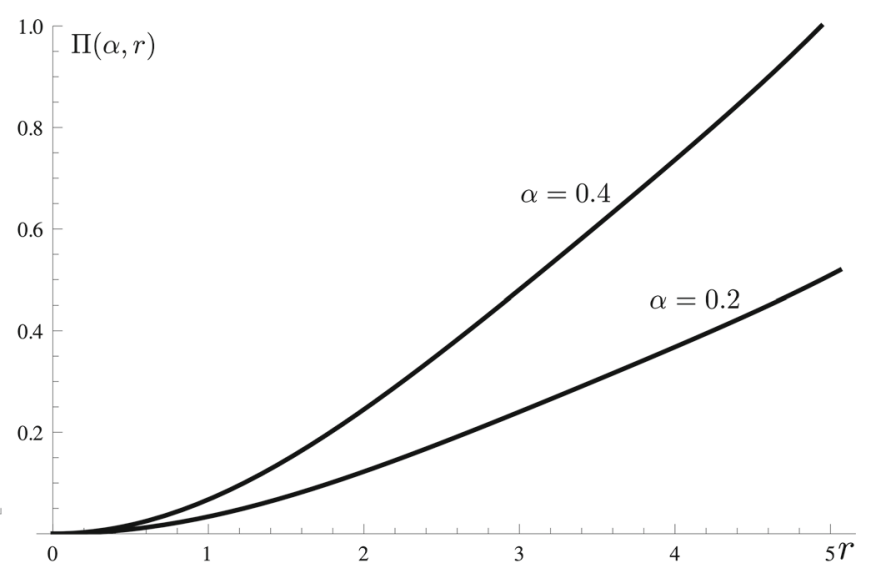

Fig. 2 Effective density $\tilde{\rho}(r)\left[10^{-3}\right]$ and anisotropy $\Pi(\alpha, r)\left[10^{-4}\right]$ for a stellar system with compactness $M / R=0.2$. Radial coordinates are in units of $M(r=5=R)$. The cosmological constant $\Lambda \sim 10^{-52}$ is too small to be relevant
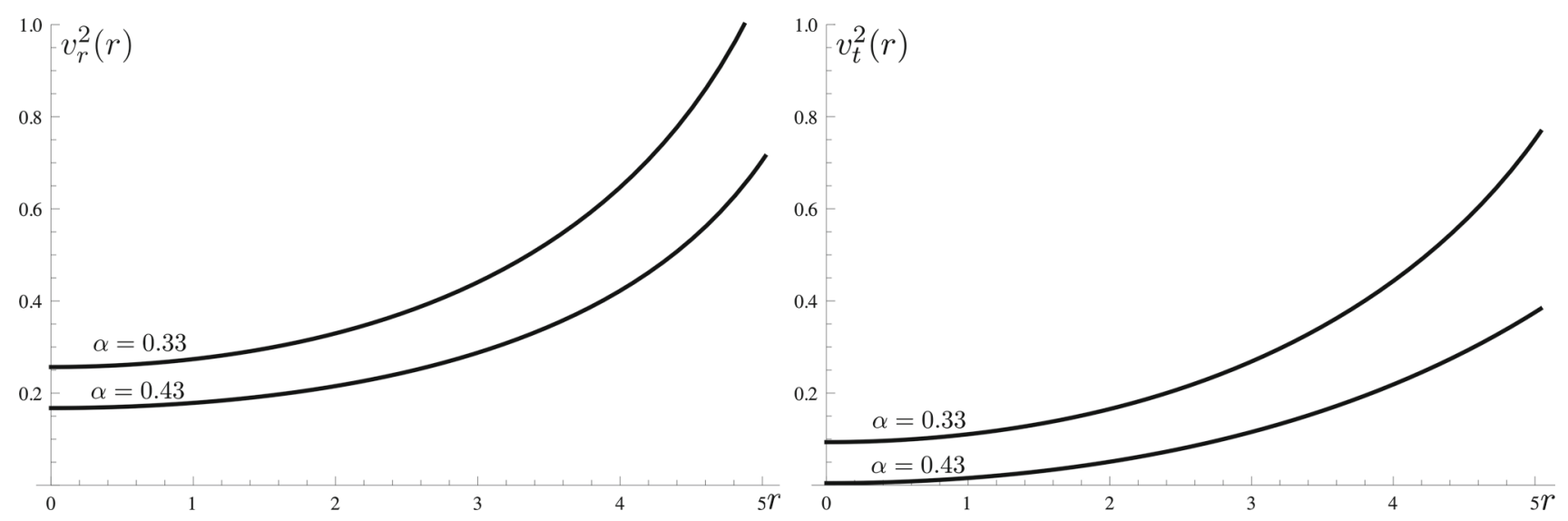

Fig. 3 Radial velocity $\left(v_{r}^{2}\right)$ and tangential velocity $\left(v_{t}^{2}\right)$ for a stellar system with compactness $M / R=0.2$. Radial coordinates are in units of $M$ $(r=5=R)$. The cosmological constant $\Lambda \sim 10^{-52}$ is too small to be relevant 
are satisfied [59]. Finally, it is worth to examine the causal conditions,

$v_{r}^{2}(r)=\frac{d \tilde{p}_{r}(r, \alpha)}{d \tilde{\rho}(r, \alpha)} \leq 1$

$v_{t}^{2}(r)=\frac{d \tilde{p}_{t}(r, \alpha)}{d \tilde{\rho}(r, \alpha)} \leq 1$,

where $v_{r}$ and $v_{t}$ are the radial and tangential sound speed, respectively, displayed in Figure 3. In both cases we observe that the speed decreases with the anisotropy and remains subluminal. We also notice that both speeds increase from the center to the stellar surface, which might be interpreted as a signal of instability. This may be true in the case of perfect fluids, however, it is not necessarily so when anisotropic effects are presents, as the stability conditions become much more involved [60,61].

\section{Conclusions}

By using the MGD-decoupling approach, we found the anisotropic and non-uniform version of the Schwarzschildde Sitter interior solution with cosmological constant given by the exact and analytical expressions displayed in Eqs. (3.1), (3.15), and (3.17)-(3.19). Contrary to the well known Schwarzchild interior solution, this new system satisfies all of the physical requirement, namely, it is regular at the origin, pressure and density are positive everywhere (at least for $\alpha>0$ ), mass and radius are well defined when the Buchdahl limit (3.9) holds, density and pressure decrease monotonically from the centre outwards (for $\alpha>0$ ), the dominant energy condition is satisfied, and the sound speeds are subluminal. Regarding this last requirement, our interior anisotropic solution is causal, showing thus that the anisotropic effects produce a more realistic stellar structure.

The matching conditions at the stellar surface were studied in detail for an outer vacuum Schwarzschild-de Siter space-time. In particular, the continuity of the second fundamental form in Eq. (2.36) was shown to yield the important result that the effective radial pressure $\tilde{p}_{r}$ can be made to vanish at the surface by a suitable choice (3.13) of the anisotropic source. The effective pressure (2.10) contains both the isotropic pressure of the undeformed Schwarzschild solution and the anisotropic effects produces by the inner geometric deformation $f^{*}$ induced by the generic energymomentum $\theta_{\mu \nu}$, which could also represent a specific matter source. If the geometric deformation $f^{*}$ is positive and therefore weakens the gravitational field [see Eq. (2.27)], an outer Schwarzschild-de Sitter vacuum could only be supported if the isotropic $p_{R}<0$ at the star surface, which can be interpreted as regular matter with a solid crust [43]. However, we could keep the standard condition $p_{R}=0$ by imposing that the anisostropic effects on the radial effective pressure be proportional to $p(r)$, as shown in Eq. (3.13). This leads to a vanishing inner deformation $f_{R}^{*}=0$ and therefore the total mass $M$ of the standard Schwarzschild interior solution is not affected by the anisotropy, as we can see in the condition (2.30). A direct consequence of this is that the surface redshift

$z=\left[1-\frac{2 M}{R}\right]^{-1 / 2}-1$

remain equal for both solutions, namely, the standard Schwarzschild solution and its new causal version.

In this paper we included a cosmological constant $\Lambda$ for generality. However, since the present value of $\Lambda$ would introduce corrections of order $10^{-52}$, its effects could be significant mainly at very large scales, with no sizeable consequences on self-gravitating stellar objects. In particular, it was shown [8] that $\Lambda$ plays a significant role for very extended polytropic spheres that could describe galactic dark matter halos (see also Ref. [62] where the effects of $\Lambda$ in several astrophysical situations is summarised). On the other hand, a large effective cosmological constant could be related to phase transitions in the early universe, and that could influence compact objects created during this period, like primordial black holes. For example, the electroweak phase transition at $T_{\text {ew }} \sim 100 \mathrm{GeV}$ corresponds to $\Lambda_{\text {ew }} \sim 0.028 \mathrm{~cm}^{-2}$, while for the quark confinement at $T_{\mathrm{qc}} \sim 100 \mathrm{MeV}$ one would have $\Lambda_{\mathrm{qc}} \sim 2.8 \cdot 10^{-10} \mathrm{~cm}^{-2}$ [63].

We conclude by mentioning that, for the study of nonprimordial compact configurations, we can safely ignore the cosmological constant without jeopardising our causal solution. This yields even simpler expressions which could be exploited more easily to investigate some interesting cases, such as the gravastar limit and the extended Kerr source [11], or even a possible generalisation including a nontrivial time dependence, as those in the exact time-dependent version found in Ref. [13], but without the space-time singularities present therein.

Acknowledgements J.O. and S.Z. have been supported by the Albert Einstein Centre for Gravitation and Astrophysics financed by the Czech Science Agency Grant No.14-37086G and by the Silesian University at Opava internal grant SGS/14/2016. J.O. thanks Luis Herrera for useful discussion and comments. L.G. acknowledges the FPI Grant BES-2014067939 from MINECO (Spain). A.S. is partially supported by Project Fondecyt 1161192, Chile and MINEDUC-UA project, code ANT 1855. R.C. is partially supported by the INFN grant FLAG and his work has also been carried out in the framework of activities of the National Group of Mathematical Physics (GNFM, INdAM) and COST action Cantata.

Data Availability Statement This manuscript has no associated data or the data will not be deposited. [Authors' comment: This is a theoretical work. No experimental data was used.]

Open Access This article is distributed under the terms of the Creative Commons Attribution 4.0 International License (http://creativecomm ons.org/licenses/by/4.0/), which permits unrestricted use, distribution, 
and reproduction in any medium, provided you give appropriate credit to the original author(s) and the source, provide a link to the Creative Commons license, and indicate if changes were made. Funded by SCOAP ${ }^{3}$.

\section{References}

1. K. Schwarzschild, Sitzungsber. Preuss. Akad. Wiss. Berlin (Math. Phys.) 1916, 189 (1916)

2. S. Weinberg, Gravitation and cosmology (Wiley, New York, 1972)

3. M.M. Som, M.A.P. Martins, A.A. Moregula, Phys. Lett. B 287, 50 (2001)

4. Z. Stuchlik, S. Hledik, J. Soltes, E. Ostgaard, Phys. Rev. D 64, 044004 (2001)

5. Z. Stuchlik, G. Torok, S. Hledik, M. Urbanec, Class. Quant. Gravity 26, 035003 (2009)

6. Z. Stuchlik, Acta Physica Slovaca 50, 219 (2000)

7. C.G. Boehmer, Gen. Rel. Grav. 36, 1039-1054 (2004)

8. Z. Stuchlik, S. Hledik, J. Novotny, Phys. Rev. D 94, 103513 (2016)

9. P.O. Mazur, E. Mottola, Proc. Nat. Acad. Sci. 101, 9545 (2004)

10. P.O. Mazur, E. Mottola, Class. Quant. Gravity 32, 215024 (2015)

11. C. Posada, Monthly Not. R. Astron. Soc. 468, 2128 (2017)

12. C. Posada, C. Chirenti, Class. Quant. Gravity 36, 065004 (2019)

13. P. Beltracchi, P. Gondolo, Phys. Rev. D 99, 084021 (2019)

14. H. Stephani, D. Kramer, M. Maccallum, C. Hoenselaers, E. Herlt, Exact solutions of Einstein's field equations (Cambridge University Press, Cambridge, 2003)

15. J. Ovalle, Phys. Rev. D 95, 104019 (2017)

16. J. Ovalle, Phys. Lett. B 788, 213 (2019)

17. J. Ovalle, R. Casadio, R. da Rocha, A. Sotomayor, Eur. Phys. J. C 78, $122(2018)$

18. L. Gabbanelli, Á. Rincón, C. Rubio, Eur. Phys. J. C 78, 370 (2018)

19. J. Ovalle, R. Casadio, R. da Rocha, A. Sotomayor, Z. Stuchlik, Eur. Phys. J. C 78, 960 (2018)

20. M. Sharif, S. Sadiq, Eur. Phys. J. C 78, 410 (2018)

21. E. Contreras, Eur. Phys. J. C 78, 678 (2018)

22. M. Sharif, S. Sadiq, Eur. Phys. J. Plus 133, 245 (2018)

23. E. Contreras, P. Bargueño, Eur. Phys. J. C 78, 558 (2018)

24. E. Morales, F. Tello-Ortiz, Eur. Phys. J. C 78, 618 (2018)

25. C. Las Heras, P. Leon, Fortsch. Phys. 66, 070036 (2018)

26. G. Panotopoulos, A. Rincón, Eur. Phys. J. C 78, 851 (2018)

27. M. Sharif, S. Saba, Eur. Phys. J. C 78, 921 (2018)

28. E. Contreras, A. Rincòn, P. Bargueño, Eur. Phys. J. C 79, 216 (2019)

29. S.K. Maurya, F. Tello-Ortiz, Eur. Phys. J. C 79, 85 (2019)

30. E. Contreras, Class. Quant. Gravity 36, 095004 (2019)
31. J. Ovalle, R. Casadio, R. da Rocha, A. Sotomayor, Z. Stuchlik, EPL 124, 20004 (2018)

32. M. Sharif, A. Waseem, Ann. Phys. 405, 14 (2019)

33. J. Ovalle, Mod. Phys. Lett. A 23, 3247 (2008)

34. J. Ovalle, Braneworld stars: anisotropy minimally projected onto the brane, In "Gravitation and Astrophysics" (ICGA9), Ed. J. Luo (World Scientific, Singapore, 2010)

35. L. Randall, R. Sundrum, Phys. Rev. Lett. 83, 3370 (1999)

36. L. Randall, R. Sundrum, Phys. Rev. Lett 83, 4690 (1999)

37. R. Casadio, J. Ovalle, R. da Rocha, Class. Quantum Gravity 32, $215020(2015)$

38. J. Ovalle, Int. J. Mod. Phys. Conf. Ser. 41, 1660132 (2016)

39. R. Casadio, J. Ovalle, Phys. Lett. B 715, 251 (2012)

40. J. Ovalle, F. Linares, Phys. Rev. D 88, 104026 (2013)

41. J. Ovalle, F. Linares, A. Pasqua, A. Sotomayor, Class. Quantum Gravity 30, 175019 (2013)

42. R. Casadio, J. Ovalle, R. da Rocha, Class. Quantum Gravity 30, 175019 (2014)

43. J. Ovalle, L.A. Gergely, R. Casadio, Class. Quantum Gravity 32 , 045015 (2015)

44. R. Casadio, J. Ovalle, R. da Rocha, EPL 110, 40003 (2015)

45. R.T. Cavalcanti, A. Goncalves da Silva, R. da Rocha, Class. Quantum Gravity 33, 215007 (2016)

46. R. Casadio, R. da Rocha, Phys. Lett. B 763, 434 (2016)

47. R. da Rocha, Phys. Rev. D 95, 124017 (2017)

48. R. da Rocha, Eur. Phys. J. C 77, 355 (2017)

49. A. Fernandes-Silva, R. da Rocha, Eur. Phys. J. C 78, 271 (2018)

50. R. Casadio, P. Nicolini, R. da Rocha, Class. Quantum Gravity 35, 185001 (2018)

51. A. Fernandes-Silva, A.J. Ferreira-Martins, R. da Rocha, Eur. Phys. J. C 78, 631 (2018)

52. A. Fernandes-Silva, A.J. Ferreira-Martins, R. da Rocha, Phys. Lett. B 791, 323 (2019)

53. L. Herrera, N.O. Santos, Phys. Rept. 286, 53 (1997)

54. M.K. Mak, T. Harko, Proc. R. Soc. Lond. A 459, 393 (2003)

55. W. Israel, Nuovo Cim. B 44, 1 (1966); Erratum-ibid B 48, 463 (1967)

56. N.O. Santos, Mon. Not. R. Astr. Soc. 216, 403 (1985)

57. C.G. Boehmer, T. Harko, Phys. Lett. B 630, 73 (2005)

58. H.A. Buchdahl, Phys. Rev. 116, 1027 (1959)

59. J. Ponce de Leon, Gen. Relat. Gravity 25, 1123 (1993)

60. L. Herrera, G.J. Ruggeri, L. Witten, ApJ 234, 1094 (1979)

61. R. Chan, L. Herrera, N.O. Santos, Monthly Not. R. Astron. Soc. 265, 533 (1993)

62. Z. Stuchlik, Mod. Phys. Lett. A 20, 561 (2005)

63. Z. Stuchlik, P. Slany, S. Hledik, Astron. Astrophys. 363, 425-439 (2000) 